\title{
In Vivo Imaging of RNA Interference
}

\author{
Hao Hong, Yin Zhang, and Weibo Cai \\ Departments of Radiology and Medical Physics and University of Wisconsin Carbone Cancer Center, University of Wisconsin-Madison, \\ Madison, Wisconsin
}

RNA interference (RNAi), an effective technique for regulating or silencing specific genes, can be applied to treat various diseases. Multiple clinical trials using RNAi are ongoing, and molecular imaging can serve as a powerful tool in RNAi-based therapies. This brief review will highlight the current progress on in vivo imaging of RNAi delivery and silencing effects. Incorporation of suitable molecular imaging techniques into future RNAibased clinical trials will provide more pieces of the puzzle, thus facilitating the transformation of RNAi into a powerful therapeutic modality in the clinic.

Key Words: RNA interference (RNAi); molecular imaging; small interfering RNA (siRNA); small hairpin RNA (shRNA); positron emission tomography (PET)

J Nucl Med 2010; 51:169-172

DOI: 10.2967/jnumed.109.066878

Since its discovery in 1998 (1), RNA interference (RNAi) has emerged as a powerful tool for therapeutic gene silencing because of its unique specificity, broad applicability, and high efficiency. Small RNAs regulate gene expression by transcriptional and posttranscriptional genesilencing mechanisms (2). The effector RNA molecules of RNAi consist of about 20-30 nucleotides, which are complexed with the RNA-induced silencing complex to generate a cascade effect, causing sequence-specific messenger RNA cleavage or translation repression (3). Using small interfering RNA (siRNA) or small hairpin RNA (shRNA), clinical applications of RNAi focus on the treatment of several diseases: age-related macular degeneration, viral infections, cancer, and neurologic disorders (2). In 2004, the first siRNA-based therapeutic (bevasiranib) entered a clinical trial for the treatment of age-related macular degeneration $(2,4)$. Currently, there are many multicenter clinical trials of RNAi ongoing around the world.

However, the clinical utility of RNAi faces several challenges. RNAi is an important endogenous regulatory mechanism in cells, and introduction of foreign siRNAs can cause undesired side effects. Certain siRNA molecules show off-target effects, and synthetic siRNAs can induce type I interferon responses and stimulate the production of inflammatory cytokines. Further-

\footnotetext{
Received Jul. 16, 2009; revision accepted Oct. 21, 2009.

For correspondence or reprints contact: Weibo Cai, Departments of Radiology and Medical Physics, University of Wisconsin-Madison, 1111 Highland Ave., Room 7137, Madison, WI 53705.

E-mail: wcai@uwhealth.org

COPYRIGHT @ 2010 by the Society of Nuclear Medicine, Inc.
}

more, efficient and specific delivery of siRNA or shRNA to target cells or organs is highly challenging (5). The ultimate goal of RNAi-based therapies will be hard to achieve without improving the biodistribution, safety, effectiveness, and reliability of gene delivery systems.

Molecular imaging techniques can serve as powerful tools for tracking siRNA or shRNA delivery in vivo, as well as for assessing the silencing effects $(6,7)$. To date, multiple molecular imaging modalities have been applied in RNAirelated research, including optical imaging (fluorescence and bioluminescence), MRI, magnetic resonance spectroscopy (MRS), SPECT, and PET (Fig. 1).

\section{IMAGING OF SIRNA DELIVERY}

The development and optimization of targeted delivery strategies (viral or nonviral) to enable efficient, systemic delivery of siRNA will be a big step forward for RNAi-based therapies. Optical imaging, radionuclide-based imaging (i.e., SPECT and PET), and MRI have been used for the assessment of various siRNA delivery techniques.

\section{Fluorescence Imaging}

Optical imaging of siRNA delivery generally adopts 2 strategies: labeling the siRNAs or labeling their carriers. An siRNA-based molecular beacon was developed for the detection and knockdown of telomerase expression in human breast cancer cells (8). The molecular beacon incorporates a fluorescence resonance energy transfer fluorophore pair (Cy3/ Cy5) and is activated upon binding to the telomerase messenger RNA in cells. Aside from excellent gene-silencing efficiency ( $\sim 80 \%$ ), the activation of the molecular beacon in cancer cells can also be visualized with fluorescence imaging. Thus, this strategy could be useful for both imaging and therapeutic applications.

Quantum dots (QDs) have been used to label siRNA carriers (mostly nanoparticles) in cell-culture studies. For example, cationic liposomes were used to deliver both QDs and siRNA to murine fibroblasts (9). The gene-silencing effect correlated directly with intracellular fluorescence (i.e., the QD signal), and more than $90 \%$ gene knockdown was achieved in the highly fluorescent cells. Subsequently, a new system was reported that incorporated a polyethylene glycol-modified QD core with siRNA and tumor-homing peptides attached (10). However, only modest gene knockdown $(<30 \%$ based on fluorescence) was achieved with this system. In another study, 
FIGURE 1. Molecular imaging techniques can be used to monitor both gene delivery efficiency and silencing effect of RNAi. In some cases, carriers can also be image tags. After further optimization and rigorous validation, many of these techniques may in future be translated into the clinic.

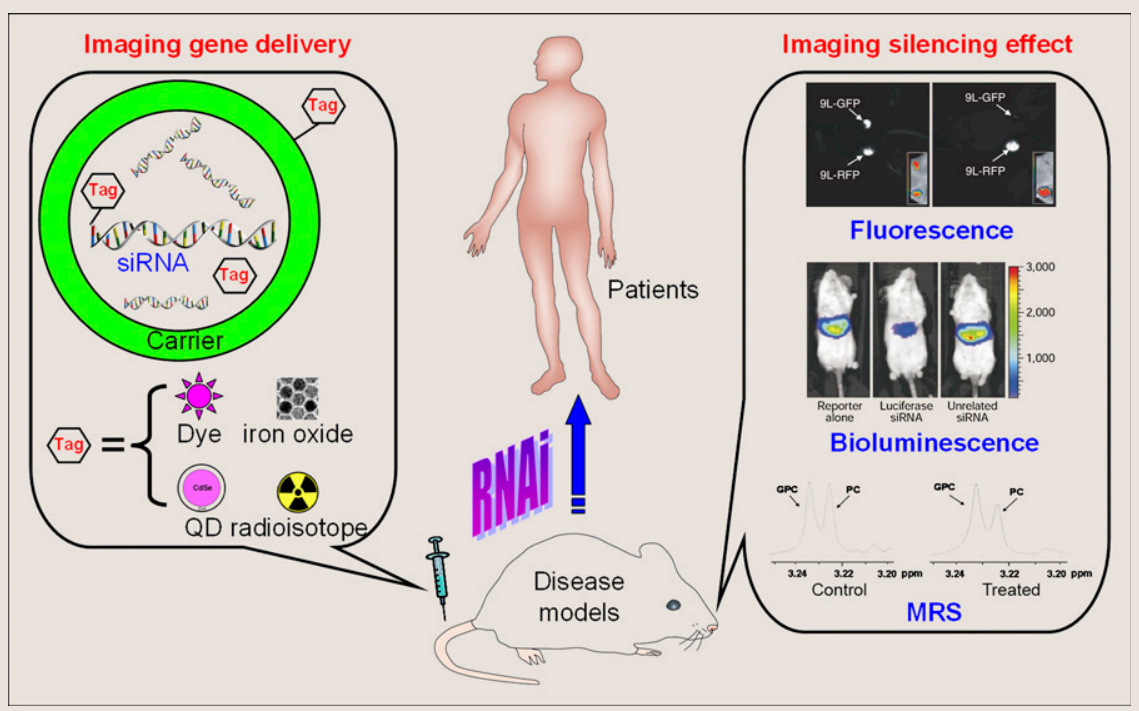

QDs were encapsulated into antibody-conjugated chitosan nanoparticles to monitor the delivery of HER2 siRNA to cancer cells (11).

Although fluorescent QDs remain attractive tools for celland animal-based studies, many barriers prevent their immediate clinical translation (12). In general, because of poor tissue penetration and lack of quantitation capability, optical imaging has limited clinical potential.

\section{Dual-Modality MRI and Fluorescence Imaging}

In 2007, a multifunctional agent was described for in vivo transfer of siRNA and simultaneous imaging of tumor targeting by MRI and near-infrared fluorescence imaging (13). The near-infrared fluorescent dye labeled magnetic nanoparticle, covalently linked to siRNA molecules specific for either model or therapeutic targets, was further modified with a membrane translocation peptide for intracellular delivery. In vivo tracking of this multifunctional agent in 2 tumor models was demonstrated by MRI and near-infrared fluorescence imaging. Further, in vivo optical imaging in the green fluorescent protein (GFP) channel confirmed that efficient gene silencing was achieved in 9L rat gliosarcoma tumors stably transfected with GFP. This study was the first example of combining noninvasive multimodality imaging and RNAi using a nanoparticle.

\section{SPECT}

The inherent characteristics of radionuclide-based imaging (e.g., sensitive, quantitative, and tomographic) make SPECT and PET more suitable for in vivo imaging of siRNA delivery than are MRI and optical imaging. ${ }^{99 \mathrm{~m}} \mathrm{Tc}$ and ${ }^{111}$ In have been used to determine the cellular delivery and biodistribution of siRNAs $(14,15)$. In addition, the stability of ${ }^{111}$ In-labeled siRNA-poly(ethyleneimine) complexes has been investigated with SPECT (16). Although these studies provided encouraging results regarding the absolute accumulation of radiolabeled siRNAs in tumors, it was not clear whether the delivered siRNA was functional and if any therapeutic effect was achieved.
PET

Because of its high sensitivity, PET has the greatest clinical potential among all molecular imaging modalities. PET and bioluminescence imaging (BLI) have been used to evaluate the biodistribution and function of siRNA-containing nanoparticles in mice inoculated with luciferase-transfected tumor cells (17). Conjugation of 1,4,7,10-tetraazacyclododecane1,4,7,10-tetraacetic acid (DOTA) to the $5^{\prime}$ end of the siRNA allowed for ${ }^{64} \mathrm{Cu}$ labeling. PET data showed that nontargeted and transferrin-targeted siRNA-containing nanoparticles exhibited similar biodistribution and tumor accumulation (17). However, BLI revealed that transferrin-targeted nanoparticles resulted in lower tumor luciferase activity than did the nontargeted nanoparticles. This strategy enabled direct and noninvasive evaluation of siRNA delivery, which can be combined with BLI to further characterize the therapeutic potential of RNAi. In future studies, certain issues such as in the vivo stability of the ${ }^{64} \mathrm{Cu}$-DOTA complex, and whether DOTA-conjugated siRNAs have RNAi efficiency comparable to that of unlabeled siRNAs, deserve further investigation.

Using the most widely applied positron emitter, ${ }^{18} \mathrm{~F}$, a recent study examined the biodistribution of radiolabeled siRNAs (18). Replacement of the $2^{\prime}$-hydroxyl group of certain nucleotides in a siRNA sequence with a fluorine atom or a methoxy group was found to be compatible with RNAi. PET, performed after intravenous injection of ${ }^{18} \mathrm{~F}$-labeled siRNAs (fluorine- or methoxy-substituted or unmodified) in rodents, showed rapid kidney and liver clearance of radioactivity. Although tissue distribution profiles of these siRNAs were similar, fluorine-modified siRNAs exhibited better blood persistence and stability than either the methoxy-substituted or the unmodified siRNAs.

The 2 strategies for imaging siRNA delivery are labeling the siRNA itself (which may affect its function or specificity and RNAi efficiency) or labeling the siRNA carrier. The latter will not affect the efficacy of siRNA, but the imaging data represent localization of the carrier rather than the siRNA. In future studies, labeling the siRNA and the carrier with different imaging tags should be investigated. Comparison of the 
biodistribution of labeled siRNA and labeled carrier will provide a more complete picture and lead to better understanding of siRNA delivery. One study described above concluded that fluorine substitution did not affect the activity of siRNAs (18); thus, optimizing ${ }^{18} \mathrm{~F}$ chemistry for siRNA labeling may provide another means for accurate evaluation of siRNA delivery.

In conclusion, molecular imaging has the potential to play a key role in selecting siRNA delivery systems with optimal biodistribution, pharmacokinetics, and targeting efficacy for future RNAi-based therapies. An alternative approach to confirm the efficiency of RNAi is to measure the silencing effect or therapeutic response. Currently, most such studies use optical imaging techniques (both bioluminescence and fluorescence), which allow semiquantitative or quantitative measurement of gene inhibition.

\section{IMAGING THE THERAPEUTIC OR SILENCING EFFECTS}

Molecular imaging of the therapeutic or silencing effects of RNAi is still at the preclinical stage, mainly through visualizing the levels of marker gene expression in living animals. These imaging markers are mostly bioluminescent enzymes, whose activity can be accurately measured on the basis of light output since background signal is minimal in animals. The use of fluorescent proteins (e.g., enhanced GFP or red fluorescent protein [RFP]) is also feasible, but tissue autofluorescence may interfere with the enhanced GFP or RFP signal.

MRS is also useful in evaluating the efficacy of RNAi-based therapies because of its potential to directly detect the biologic outcomes. By measuring the chemical shifts of certain compounds (e.g., choline, whose concentration in tissues or tumors can be altered by certain therapeutic intervention), MRS may have better clinical utility than does optical imaging.

\section{BLI}

Many proof-of-principle studies on in vivo RNAi adopted the strategy of silencing luciferase expression as a measurement of siRNA or shRNA activity $(19,20)$. In several recent studies, BLI has been used to investigate the dose or time dependence of vector-based RNAi (21) and to optimize various siRNA delivery methods $(22,23)$.

Multidrug resistance (MDR), a major obstacle for successful chemotherapy of cancer, can be caused by overexpression of P-glycoprotein, a product of the $M D R I$ gene. In an elegant study, BLI was used for noninvasive assessment of P-glycoprotein silencing (24). Taking advantage of the fact that the substrate of Renilla luciferase (i.e., coelenterazine) is effluxed out of the cells by P-glycoprotein, the investigators used BLI to evaluate the shRNA-mediated downregulation of P-glycoprotein activity in both cultured cells and tumor implants in living animals (24). Further, a similar strategy was also shown to be applicable to firefly luciferase (24).

In another report, the role of hypoxia-inducible factor- $1 \alpha$ in glioma growth was investigated in vivo with RNAi (25). BLI revealed that RNAi therapy could significantly attenuate glioma growth by reducing hypoxia-inducible factor-1 $\alpha$ levels constitutively (using shRNA) or transiently (using siRNA).
Despite the success of BLI in evaluating the gene-silencing efficiency of RNAi, such data should be interpreted with caution because firefly luciferase has a relatively short half-life in living cells (a few hours). In contrast, fluorescent proteins have much longer half-lives (up to $26 \mathrm{~h}$ ). Therefore, fluorescence imaging with enhanced GFP or other fluorescent proteins can be more advantageous than BLI in evaluating the long-term effects of RNAi.

\section{Fluorescence Imaging}

Enhanced GFP and its variants have been primarily used for ex vivo imaging studies to monitor the efficiency of RNAi. Many of these studies provided answers to important questions such as the subcellular distribution and bioavailability of siRNAs delivered by various formulations, including tumortargeted carriers. In several of the studies mentioned above, enhanced GFP was used to evaluate the silencing effect of siRNAs and confirm their delivery to the target organs $(10,13)$. In another report, the effect of intratumoral injection of siRNA (against enhanced GFP) into enhanced GFP-expressing B16F10 melanoma tumors, followed by application of an external electric field, was evaluated with fluorescence imaging (26). A significant decrease in tumor enhanced GFP fluorescence was observed by in vivo imaging within $2 \mathrm{~d}$ after treatment.

\section{MRS}

MRS, with unique specificity but low sensitivity, can be used for imaging the metabolic changes that accompany many diseases such as cancer. Elevated levels of phosphocholine and total choline metabolites are well-established characteristics of many cancer cells; thus, monitoring them with MRS can be effective in assessing the therapeutic effect of RNAi. Recently, a breast cancer model was used to investigate lentiviral vector-mediated shRNA downregulation of choline kinase, the enzyme that converts choline to phosphocholine (27). After intravenous injection of lentiviruses (which express the shRNA against choline kinase) into MDA-MB-231 tumor-bearing mice, noninvasive ${ }^{31} \mathrm{P}$ MRS revealed that phosphocholine and phosphomonoester levels in the tumor significantly decreased. More importantly, choline kinase silencing resulted in reduced tumor growth and proliferation. This study proved the principle that noninvasive MRS can be used to monitor RNAibased therapies with high accuracy.

\section{CONCLUSION AND FUTURE PERSPECTIVES}

A variety of molecular imaging techniques have been explored for in vivo imaging of RNAi (Table 1). Clearly, continued development of noninvasive imaging strategies for monitoring both siRNA/shRNA delivery and its gene-silencing effect will provide more insights into RNAi-based therapies. Optical techniques used to be the only tools for noninvasively evaluating the effects of RNAi. Although useful in preclinical settings, the clinical potential of these techniques is limited. MRS, recently applied to RNAi-based therapies, has certain clinical potential but may be limited by its sensitivity. Imaging siRNA delivery with SPECT/PET is clinically relevant, but the labeling chemistry needs to be chosen carefully. Much future effort will be 
TABLE 1. Brief Summary of In Vivo Imaging of RNAi

\begin{tabular}{|c|c|c|c|c|c|}
\hline Modality & $\begin{array}{l}\text { Imaging delivery } \\
\text { or effect? }\end{array}$ & Image tag & $\begin{array}{l}\text { Labeling gene } \\
\text { or carrier? }\end{array}$ & $\begin{array}{l}\text { Clinical } \\
\text { potential }\end{array}$ & Reference \\
\hline Fluorescence & Both & Fluorescent dye, QD, GFP, etc. & Both & + & $8-11,13,26$ \\
\hline MRI & Delivery & Iron oxide & Carrier & ++ & 13 \\
\hline SPECT & Delivery & $99 \mathrm{mTc}$ and ${ }^{111} \mathrm{In}$ & Both & +++ & $14-16$ \\
\hline PET & Delivery & ${ }^{18} \mathrm{~F}$ and ${ }^{64} \mathrm{Cu}$ & Gene & +++ & 17,18 \\
\hline BLI & Effect & Luciferases & NA & None & $17,20-25$ \\
\hline MRS & Effect & Chemical shift & NA & ++ & 27 \\
\hline
\end{tabular}

required to optimize various imaging techniques for use in RNAibased therapies.

The most significant barrier to the widespread use of RNAi in the clinic is delivery. Solving this problem will require the development of clinically suitable, safe, and effective genedelivery systems. Incorporation of molecular imaging techniques to monitor the gene-delivery efficiency or the silencing effect, which is missing from most of the currently ongoing RNAi-based clinical trials, may dramatically facilitate the transformation of RNAi into a powerful therapeutic modality in the clinic.

Over the last decade, molecular imaging with PET and SPECT has advanced dramatically, and many PET and SPECT probes are already in clinical trials for a wide variety of targets $(28,29)$. Some of these probes may be directly used to monitor the therapeutic effect of RNAi in the future, if the imaging target is related to the target of RNAi. Lastly, monitoring RNAi-based therapies (both gene delivery and the silencing effect) with a single imaging modality may not be sufficient in many cases. Rational design and use of dual-modality or multimodality imaging in future RNAi-related studies will allow researchers to acquire more pieces of the puzzle, thereby fulfilling the enormous potential of RNAi.

\section{ACKNOWLEDGMENTS}

We are grateful for financial support from the Wisconsin Partnership Program, UW Carbone Cancer Center, NCRR 1UL1RR025011, and Susan G. Komen for the Cure.

\section{REFERENCES}

1. Fire A, Xu S, Montgomery MK, Kostas SA, Driver SE, Mello CC. Potent and specific genetic interference by double-stranded RNA in Caenorhabditis elegans. Nature. 1998;391:806-811.

2. Castanotto D, Rossi JJ. The promises and pitfalls of RNA-interference-based therapeutics. Nature. 2009;457:426-433.

3. Grimm D. Small silencing RNAs: state-of-the-art. Adv Drug Deliv Rev. 2009; 61:672-703.

4. Shen J, Samul R, Silva RL, et al. Suppression of ocular neovascularization with siRNA targeting VEGF receptor 1. Gene Ther. 2006;13:225-234.

5. Whitehead KA, Langer R, Anderson DG. Knocking down barriers: advances in siRNA delivery. Nat Rev Drug Discov. 2009;8:129-138.

6. Bogdanov AA Jr. Merging molecular imaging and RNA interference: early experience in live animals. $J$ Cell Biochem. 2008;104:1113-1123.

7. Moore A, Medarova Z. Imaging of siRNA delivery and silencing. Methods Mol Biol. 2009:487:93-110.

8. Chang E, Zhu MQ, Drezek R. Novel siRNA-based molecular beacons for dual imaging and therapy. Biotechnol J. 2007;2:422-425.
9. Chen AA, Derfus AM, Khetani SR, Bhatia SN. Quantum dots to monitor RNAi delivery and improve gene silencing. Nucleic Acids Res. 2005;33:e190.

10. Derfus AM, Chen AA, Min DH, Ruoslahti E, Bhatia SN. Targeted quantum dot conjugates for siRNA delivery. Bioconjug Chem. 2007;18:1391-1396.

11. Tan WB, Jiang S, Zhang Y. Quantum-dot based nanoparticles for targeted silencing of HER2/neu gene via RNA interference. Biomaterials. 2007;28:1565-1571.

12. Cai W, Hsu AR, Li ZB, Chen X. Are quantum dots ready for in vivo imaging in human subjects? Nanoscale Res Lett. 2007;2:265-281.

13. Medarova Z, Pham W, Farrar C, Petkova V, Moore A. In vivo imaging of siRNA delivery and silencing in tumors. Nat Med. 2007;13:372-377.

14. Liu N, Ding H, Vanderheyden JL, Zhu Z, Zhang Y. Radiolabeling small RNA with technetium-99m for visualizing cellular delivery and mouse biodistribution. Nucl Med Biol. 2007;34:399-404.

15. Merkel OM, Librizzi D, Pfestroff A, Schurrat T, Behe M, Kissel T. In vivo SPECT and real-time gamma camera imaging of biodistribution and pharmacokinetics of siRNA delivery using an optimized radiolabeling and purification procedure. Bioconjug Chem. 2009;20:174-182.

16. Merkel OM, Librizzi D, Pfestroff A, et al. Stability of siRNA polyplexes from poly(ethylenimine) and poly(ethylenimine)-g-poly(ethylene glycol) under in vivo conditions: effects on pharmacokinetics and biodistribution measured by fluorescence fluctuation spectroscopy and single photon emission computed tomography (SPECT) imaging. J Control Release. 2009;138:148-159.

17. Bartlett DW, Su H, Hildebrandt IJ, Weber WA, Davis ME. Impact of tumorspecific targeting on the biodistribution and efficacy of siRNA nanoparticles measured by multimodality in vivo imaging. Proc Natl Acad Sci USA. 2007;104: 15549-15554.

18. Viel T, Boisgard R, Kuhnast B, et al. Molecular imaging study on in vivo distribution and pharmacokinetics of modified small interfering RNAs (siRNAs). Oligonucleotides. 2008;18:201-212.

19. Kurreck J. RNA interference: from basic research to therapeutic applications. Angew Chem Int Ed Engl. 2009;48:1378-1398.

20. McCaffrey AP, Meuse L, Pham TT, Conklin DS, Hannon GJ, Kay MA. RNA interference in adult mice. Nature. 2002;418:38-39.

21. Kobayashi N, Matsui Y, Kawase A, et al. Vector-based in vivo RNA interference: dose- and time-dependent suppression of transgene expression. J Pharmacol Exp Ther. 2004;308:688-693.

22. Hassani Z, Lemkine GF, Erbacher P, et al. Lipid-mediated siRNA delivery downregulates exogenous gene expression in the mouse brain at picomolar levels. $J$ Gene Med. 2005;7:198-207.

23. Dassie JP, Liu XY, Thomas GS, et al. Systemic administration of optimized aptamer-siRNA chimeras promotes regression of PSMA-expressing tumors. Nat Biotechnol. 2009;27:839-846.

24. Pichler A, Zelcer N, Prior JL, Kuil AJ, Piwnica-Worms D. In vivo RNA interference-mediated ablation of MDR1 P-glycoprotein. Clin Cancer Res. 2005;11:4487-4494.

25. Gillespie DL, Whang K, Ragel BT, Flynn JR, Kelly DA, Jensen RL. Silencing of hypoxia inducible factor-1alpha by RNA interference attenuates human glioma cell growth in vivo. Clin Cancer Res. 2007;13:2441-2448.

26. Golzio M, Mazzolini L, Ledoux A, et al. In vivo gene silencing in solid tumors by targeted electrically mediated siRNA delivery. Gene Ther. 2007;14:752-759.

27. Krishnamachary B, Glunde K, Wildes F, et al. Noninvasive detection of lentiviral-mediated choline kinase targeting in a human breast cancer xenograft. Cancer Res. 2009;69:3464-3471.

28. Cai W, Chen X. Multimodality molecular imaging of tumor angiogenesis. J Nucl Med. 2008;49(suppl 2):113S-128S.

29. Cai W, Niu G, Chen X. Multimodality imaging of the HER-kinase axis in cancer. Eur J Nucl Med Mol Imaging. 2008;35:186-208. 